\title{
New Technologies of Power Transistors for Efficiency Increase of Power Converters: The Reliability Consideration
}

\author{
Pascal Dherbécourt ${ }^{1}$, Ahmed El Oualkadi ${ }^{2}$, Eric Joubert $^{1}$, Al Mehdi Bouchour ${ }^{1,2}$, \\ Wadia Jouha ${ }^{2}$, Mohamed Masmoudi ${ }^{1}$, Olivier Latry ${ }^{1}$ \\ \{pascal.dherbecourt@univ-rouen.fr, eloualkadi@gmail.com\} \\ ${ }^{1}$ Groupe de Physique des Matériaux UMR 6634, Normandie University, UNIROUEN, \\ INSA Rouen, CNRS. Avenue de l'université B.P 12, Saint Etienne du Rouvray, France \\ ${ }^{2}$ Laboratory of Information Technology and Communication, National School of Applied \\ Sciences of Tangier. University Abdelmalek Essaadi, Tétouan, Maroc
}

\begin{abstract}
This paper proposes a methodology to study the reliability and failure analysis of new technologies of power transistors. The use of wide gap materials such as Silicon Carbide $(\mathrm{SiC})$ and Gallium Nitride $(\mathrm{GaN})$ is now a good alternative to meet the integration requirements of energy conversion systems. However, the reliability of these components is a crucial issue. Aging in operating conditions is considered, associated with electrical measurements to highlight nondestructive degradations of the performance of studied transistors. A step of looking for failure mechanisms in the material is made in order to identify any physical degradation. The information collected provide the user with valuable data and help to make an optimum choice of the component which can be integrated into the equipment. The presented work proposes the description of a methodology that meets these requirements and shows the study of two technologies of power transistors used in new generations of power converters.
\end{abstract}

Keywords: Electronic reliability, failure analysis, power transistor, ageing tests.

\section{Introduction}

The total market for Silicon Carbide (SiC) power components will account for more than one billion US dollars (US \$ 1 trillion) by Yole Development organization in 2022 [1]. Applications such as PFC / power supplies and photovoltaic inverters are now contributing to the growth of this market. Photovoltaic applications seem to largely integrate $\mathrm{SiC}$ products. Indeed, SiC solutions offer a better system performance / cost ratio for photovoltaic inverters. In the future, applications related to electric vehicles, rail and others will also contribute to the evolution of the market.

In this context, the new power transistor technologies, now available on the market, offer increased performance compared to traditional silicon components, in terms of high voltage, high temperature and high switching frequencies.

However, these new wide gap components show random degradation problem, and reliability studies are still needed to qualify these components [2], [3], [4], [5]. The approach by long-term testing under operating conditions remains essential for integrators. 
The components under test are then aged thanks to dedicated benches where the electrical and environmental measurements are monitored. Degradations are observed and recorded, non-destructive and microstructural analyzes are made in order to identify the failure mechanisms responsible for performance degradations.

This set of techniques is implemented in the GPM laboratory, for applications of microwave amplifiers and switching power transistors, the work is carried out in collaboration with academic and industrial partners from the aeronautics, automotive and renewable energy sectors [6], [7], [8], [9].

In this paper, we present the general methodology developed in collaboration with the research team and partners meeting the defined objectives. Afterwards, we develop two examples of studies which are based on SiC MOSFET (Metal Oxide Semiconductor Field Effect Transistors) and GaN power transistors for switching applications.

\section{Reliability and failure analysis study procedure of next-generation power transistors}

The proposed methodology is summarized in Figure 1, which provides an overview of the various stages of the study of reliability and physical failure analysis of wide gap technology transistors. The studied components come mostly from the commercial sector and, thereby, little prior knowledge is available concerning the operation and physical structure of these devices. The first step, identified as step 1, consists in searching, from the manufacturer's documents or data sheets, the electrical, physical, and structural characteristics of the DUT.

From the data collected, failure indicators are defined, and electrical characterization based on measurements are implemented by means of the development of specific benches, this is step 2.

In step 3, the operational conditions are established, the component is aged on the dedicated bench, according to a particular mission profile, the electrical parameters are monitored, and measurements of failure indicators are regularly performed after each aging phase. A specific bench equipped with current measuring probe up to $30 \mathrm{~A}$ is used to record the I-V static characteristics of the transistor under test, a commercial source meter is used to measure both low gate and drain leakage currents.

According to one or more criterion of variations of the measured parameters, typically a variation of $20 \%$ of the nominal value, the aging phase is stopped, and the step 4 then consists in decapsulating the resin or ceramic packaging by laser ablation and chemical etching. This delicate operation, must be performed in order to maintain the integrity of the component. The active chip of the component is then accessible for a visual investigation, then by optical microscopy, the extrinsic elements such as metal access surfaces and bonds are controlled.

In order to precisely locate a possible defect, the PEM (Photon Emission Microscopy) and Optical Beam Induced Resistance Change (OBIRCH) techniques complete the analysis at this stage. Step 5 is implemented with a level of investigation depending on the requirements of the study. Scanning or transmission electron microscopy tools can be used, associated with a 
FIB tool (Focus Ion Beam) to explore the internal structure of the component at the ultimate scale of a few tens of nanometers.

\section{Electrical characterization \\ Instrumentation and ageing in operating conditions}
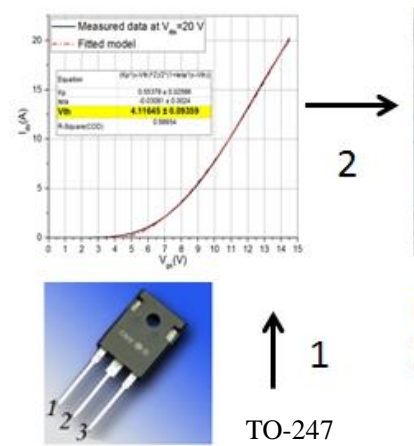

TO-247

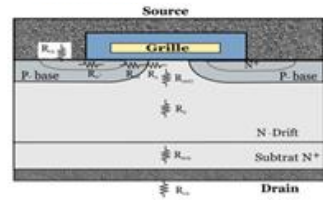

Study of component characteristics

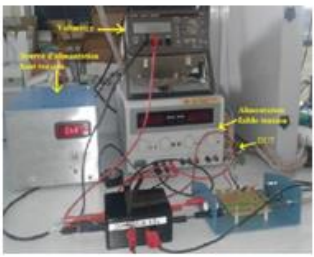

Probability study of reliability law Weibull,..

3

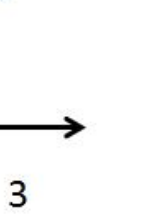

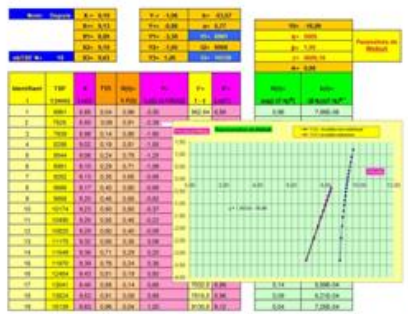

Laser or chemical decapsulation

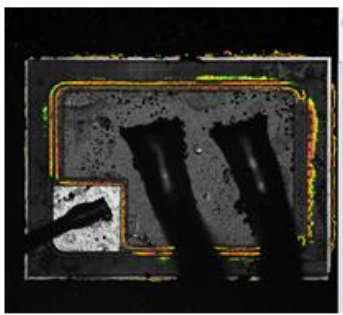

PEM or $\mathrm{OBIRCH}$

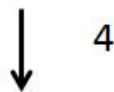

Microstructural Analysis FIB, MET, SAT

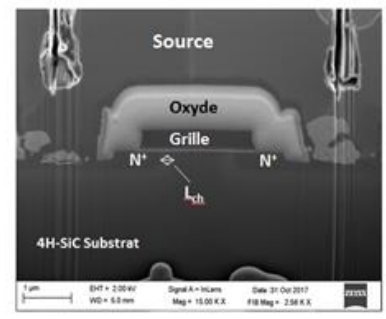

Fig. 1. Methodology to study reliability in operational conditions.

In order to meet the users requirements, study of reliability using Weibull law can be implemented in the methodology. The different parameters: Failure rate (Lambda), MTBF (Mean Time Between Failures) and MTTR (Mean Time To Repair) provide valuable information for system design and maintenance. The reference [10] describes reliability studies carried out on RFID tags, the performance of two tags of two manufacturers aged in temperature storage were compared.

These various steps make it possible to validate the study of the component in its mission profile, thus meeting the requirements of the user, but also to extract and analyze the physical defects at the origin of degradations of its characteristics. In the following paragraphs, we will develop examples of studies for new generations of SiC MOSFET and GaN power transistors, using the methodology described above. 


\section{Reliability and physical analysis of SiC power MOSFET failures for switching applications}

Due to their interesting characteristics in thermal and in high level voltage domains, $\mathrm{SiC}$ MOSFETs are a promising technology. However, the feedback on their reliability is not as important as an older technology like Si, especially concerning the impact of ESD (Electro Static Discharge). This stress is important since the specific structure of SiC MOSFET may be sensitive to this kind of stress, and because electrostatic discharges are present at several places in the context of a power transistor.

The ESD appears when two objects with different electrostatic potentials get closer fast and generates a fast and important transfer of charges. This may also cause irreversible damage at manufacturing, handling or storage of an electronic device or component. The microelectronics industries are very concerned about this subject and are constantly looking for solutions, such as protection circuits, to control it and limit its consequences. An ESD test is also important to characterize the immunity of a transistor or a technology regarding this stress.

Several models of ESD have been developed and considered various contexts of stress. At first Human Body Model (HBM) simulates the electrical behavior of an operator standing in front of the table where the device is located and touching it with a finger [11]. This model is the most used since human is the main cause of ESD in industry. Usually, this simulation is achieved with an adapted couple resistor/capacitance. The Machine Model (MM) simulates the discharge produced by accumulation in a machine on a component [12]. This discharge is stronger than HBM since the electrical resistance of a machine is often very low. At least, Charged Device Model (CDM) considers a charge accumulated by the component itself during storage or manipulation confronted to a fixed potential of an external object (ground) [13].

For each discharge model, different tests protocols may be used in accordance with specific industrial standards like MIL-HDK, JEDEC, AEC, among others. For example, the ISO 10605:2008 describes tests to evaluate performances of electronic modules in road automotive context. This standard considers ESD appearing during assembly, due to maintenance staff or due to user. The tests in the ISO 10605 standard, which often involve an ESD gun comprising a R/C cell, use either a $330 \Omega$ or $2 \mathrm{k} \Omega$ resistance, depending on the use of a metallic tools or not by user [14]. Several levels of severity are fixed by voltage from $2 \mathrm{kV}$ to $15 \mathrm{kV}$.

When a MOSFET undergoes an ESD, a strong current is flowing during a few nanoseconds, which causes an important and local rise of temperature and various kinds of failures in the transistor structure [15], [16]:

- Filamentation: appearance of a conducting filament between source and drain

- Oxide breakdown: the gate oxide is destroyed by an overvoltage

- Hot carrier injection: nondestructive but modifies the isolating layers and the electrical characteristics of the transistor.

- Bonding breakdown: an overcurrent destroys the bondings by fusion. 
The ESD stress implies also important consequences of the SiCMOSFET technology and test campaigns have to be realized to evaluate its impacts on a specific transistor family. The following parts present such a campaign on two generations of transistors of the same manufacturer, to understand evolution of ESD impact between them.

\subsection{SiC MOSFETs under test}

Among the leaders in SiC technology, the manufacturer Wolfspeed commercialized three generations of power transistors since 2011. Each generation improves the maximum drain current and the maximal case temperature, but valuable information is unavailable concerning ESD immunity under specific conditions.

Table 1. References of components involved in this study.

\begin{tabular}{lllll}
\hline Generation & Ref. & $\mathrm{I}_{\mathrm{ds}}(\max )$ & $\mathrm{V}_{\mathrm{ds}}(\max )$ & $\mathrm{Tc}(\max )$ \\
\hline Gen2L & $\mathrm{C} 2 \mathrm{M} 0280120 \mathrm{D}$ & $10 \mathrm{~A}$ & $1200 \mathrm{~V}$ & $150^{\circ} \mathrm{C}$ \\
Gen2H & $\mathrm{C} 2 \mathrm{M} 0160120 \mathrm{D}$ & $19 \mathrm{~A}$ & $1200 \mathrm{~V}$ & $150^{\circ} \mathrm{C}$ \\
Gen3L & $\mathrm{C} 3 \mathrm{M} 0280090 \mathrm{D}$ & $11,5 \mathrm{~A}$ & $900 \mathrm{~V}$ & $150^{\circ} \mathrm{C}$ \\
Gen3H & $\mathrm{C} 3 \mathrm{M} 0120090 \mathrm{D}$ & $23 \mathrm{~A}$ & $900 \mathrm{~V}$ & $150^{\circ} \mathrm{C}$ \\
\hline
\end{tabular}

The following study involves the two last Wolfspeed generations. In table 1, letters L and $\mathrm{H}$ are indicating "Low" or "High" current transistors. In each generation, different references have various electrical characteristics and different power application. All these transistors are based on a VDMOS structure which is shown in Figure 2.

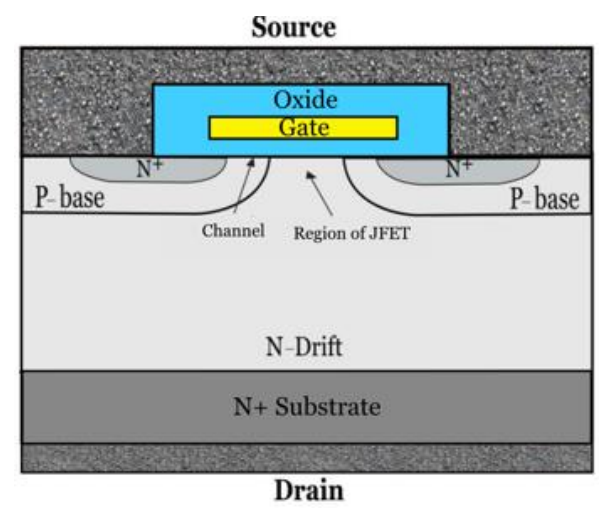

Fig. 2. Schematic structure of Wolfspeed transistors

\subsection{ESD Tests}

The ESD tests were realized on 8 components of 4 categories ( 2 of each) of Table. 1 respecting ISO 10605 standard. An electrostatic gun shown in Figure 3 was used with a couple $2 \mathrm{k} \Omega / 330 \mathrm{pF}$ simulating an HBM discharge model. 


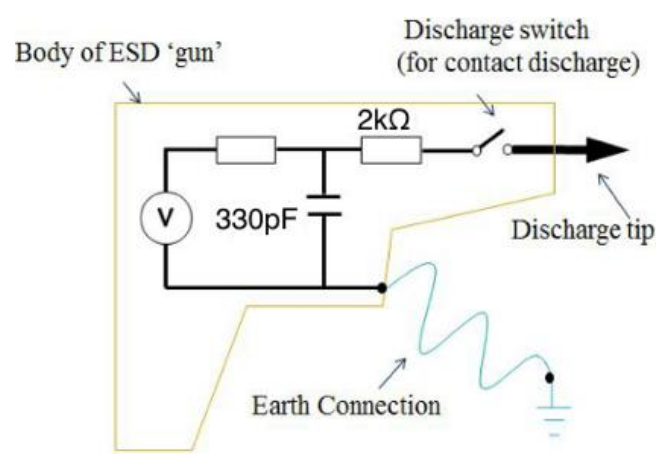

Fig.3. Electrostatic Gun schematic

Stress of $8 \mathrm{kV}$ and $15 \mathrm{kV}$ were applied repeatedly (4 times and up to 12 times on some transistors) on gates pin to evaluate gate oxide robustness and to appreciate evolutions between generations. Drains and sources pin were leaved open.

\subsection{Results and analysis}

Electrical measurements have been achieved before and after stress in order to estimate discharge impact on the behavior of the transistors. This comparison yields interesting information concerning the failures that may appear in the devices.

At first, saturation current $\mathrm{I}_{\mathrm{dsat}}$ and on-state resistance $\mathrm{R}_{\mathrm{ds}(\mathrm{on})}$ are determined from the output characteristics $\left(I_{\mathrm{ds}}=\mathrm{f}\left(\mathrm{V}_{\mathrm{ds}}\right)\right)$ of the transistors. These parameters show few variations due to the stress $\left(\mathrm{R}_{\mathrm{ds}(\mathrm{on})}\right.$ decreases and $\mathrm{I}_{\mathrm{dsat}}$ increases, about $1 \%$ for both) on two generations except for a transistor Gen3L that received a $15 \mathrm{kV}$ stress where $I_{d s}$ decreases and $R_{d s(o n)}$ increases about $10 \%$ for both.

Leakage currents $I_{g s s}$ and $I_{d s s}$ were measured on a specific bench and schematic. $I_{g s s}$ increases a lot for both generations (average values about 3000\% for Gen2 and 10000\% for Gen3). $\mathrm{I}_{\mathrm{dss}}$ increases about $100 \%$ for Gen2L and Gen3L but doesnot change significantly for Gen2H and Gen3H.

Threshold voltage $V_{t h}$, transconductance $K_{p}$ and transverse electrical field $\theta$ were determined from output characteristics with a Levenberg-Marquardt Algorithm [9]. The stress induces an average decrease of $2 \%$ for $\mathrm{V}_{\text {th }}$ on both generations, with highest values on Gen3L transistors. $K_{p}$ and $\theta$ increase slightly for both generations.

At least, $\mathrm{C}-\mathrm{V}$ measurements on gate-source, drain-source and drain-gate diode were performed on a specific bench, to get more information on failure process during the stress. The measured capacitance variations globally reveal the appearance of positive traps in the oxide volume. The effect of these traps is in good accordance with the decrease of $\mathrm{V}_{\text {th }}$ and the increase of $I_{g s s}$.

All the variations observed yields to a conclusion that the ESD stress mainly affects the oxide volume near the gate of the transistors and also the input parameters of the transistor. 
High current transistors (Gen2H and $\mathrm{Gen} 3 \mathrm{H})$ are less sensitive to this stress and no significant difference seems to appear between the two generations.

\section{Switching GaN transistor for energy conversion applications.}

The GaN technology is another alternative to $\mathrm{SiC}$ for increasing the integration of energy conversion devices. Thanks to these new devices, the automobile market anticipates an integration density multiplied by 3 , with a loss reduction of $50 \%$ and a cost reduction of $20 \%$ [17]. The advantage of the GaN technology compared to silicon substrate results from the ability to operate at: high frequency due to increased velocity saturation, high temperature and high voltage due to the large band gap material [18], [19]. In this paper, we use anew commercial component from GaN systems manufacturer, whose reference is GS66508P, it is a p-type gate normally-off which operates in the 650V/30A range, its main characteristics are given in reference [20].

\subsection{Aging tests with switching mode}

The successive aging steps and the dynamic measurements are carried out by means of a dedicated switching bench described in reference [9]. Figure 4a. shows a view of this setup. This includes two transistors noted $\mathrm{DUT}_{\mathrm{C}}$ and $\mathrm{DUT}_{\mathrm{V}}$ switching the DC source voltage to fix the desired electrical parameters in two states. The $\mathrm{DUT}_{\mathrm{M}}$ is alternately subjected to a high current by the mean of DUT $\mathrm{C}_{\mathrm{C}}$, then, out of conduction, subjected to an electric field by $\mathrm{DUT}_{\mathrm{M}}$.

The aging bench eliminates the need for significant energy consumption because, with this original operating principle, no power load is needed. To ensure this operation, the $\mathrm{PWM}_{1}$ and $\mathrm{PWM}_{2}$ signals are two complementary pulses whose pulse width can be modulated. The bench is instrumented using a digital oscilloscope connected to measurement probes.

Also, it places similar electrical waveforms on $\mathrm{DUT}_{\mathrm{M}}$, which could exist in a large class of power management products such as DC-DC power converters. Moreover, it enables dynamic measurements, like: dynamic resistance $\left(R_{d y n}\right)$ and switching times (tr and $\left.t f\right)$.

The DUT $_{M}$ current and voltage experiment waveforms are shown in Figure. 4b. As can be observed, $\mathrm{V}_{\mathrm{DS}}$ switch from $1.14 \mathrm{~V}$ to $24 \mathrm{~V}$ with a rise time equals to $53.46 \mathrm{~ns}$ and $\mathrm{I}_{\mathrm{DS}}$ switch from $0 \mathrm{~A}$ to $14 \mathrm{~A}$ with a rise time equals to $3.94 \mu \mathrm{A}$. The studied switching conditions are chosen so that the DUT $_{M}$ switches in its Safe Operating Area (SSOA).

\subsection{Degradation detection and measurements caused by the aging phase}

The studied component is aged in operating conditions with the bench described in Figure 4a. At regular time intervals, measurements are made to assess the evolution of some relevant and significant electrical parameters. To meet this goal, the threshold voltage $V_{\text {th }}$ is extracted using the Extrapolation in the Linear Region (ELR) method. The transconductance is defined as the maximum first derivative of the input characteristic in the saturation region, and 
the on-state $\mathrm{R}_{\mathrm{DS}(\mathrm{ON})}$ is defined as the inverse of the slope of output characteristics in the linear region.

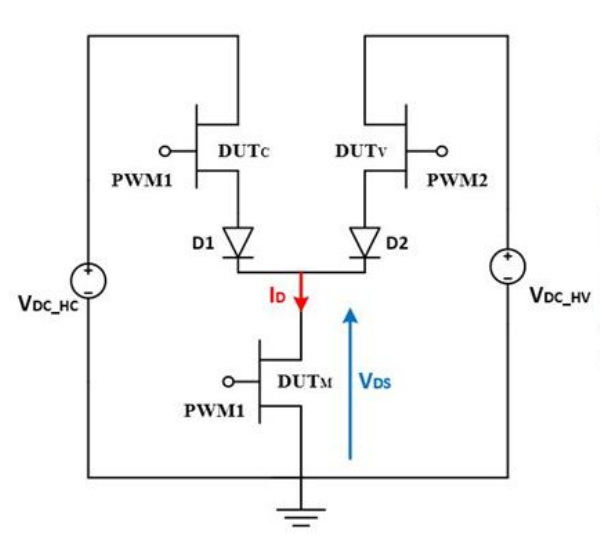

(a)

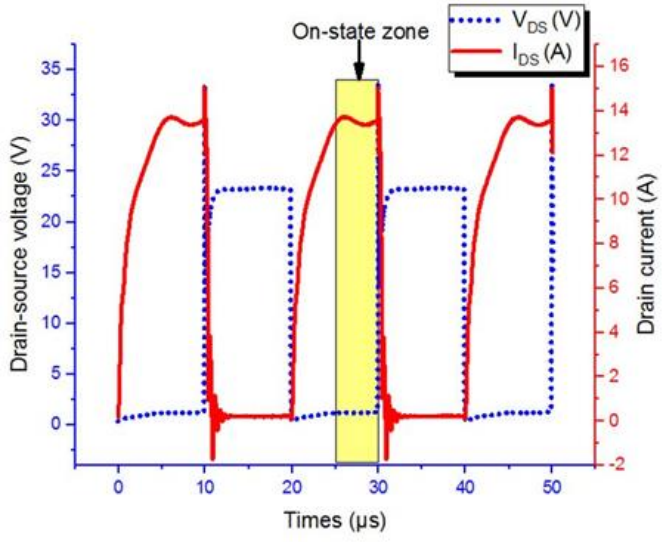

(b)

Fig. 4. (a) Setup for ageing the device in operational conditions. (b) Chronograms for $I_{D S}$ and $\mathrm{V}_{\mathrm{DS}}$ measurements.

The switching frequency is set at $50 \mathrm{kHz}$ with a duty cycle of the control signal equals to $50 \%$. The study component is thus aged at the limited Safe Operating Area (SSOA) for more than 1000 hours in operating conditions on the dedicated bench.

To ensure that the aging of the $\mathrm{DUT}_{\mathrm{M}}$ respects the SSOA, the measurements of the junction temperature $T_{J}$ and the dynamic $R_{D S(O N)}$ are performed, the temperature $T_{J}$ is estimated using the thermal model and thermal Ohm's law of DUT $_{M}$ package mounted on a PCB and a heat sink.

From experimental measurements chronograms, the power loss $\left(\mathrm{P}_{\text {loss }}\right)$ is calculated which is equal to $16.68 \mathrm{~W}$, the corresponded $\mathrm{T}_{\mathrm{J}}$ is calculated equal to $86{ }^{\circ} \mathrm{C}$ which is blow the allowable $\mathrm{T}_{\mathrm{J}}=150^{\circ} \mathrm{C}$ from the data sheet.

Table 2 summarize the values of $\mathrm{R}_{\mathrm{DS}(\mathrm{ON})}, \mathrm{g}_{\mathrm{m}}$ and $\mathrm{I}_{\mathrm{D}}$ before and after 1008 hours of aging $\mathrm{DUT}_{\mathrm{M}}$ under the switching conditions.

Table 2. 1008 hours aging results for the DUT.

\begin{tabular}{llll}
\hline & $\mathrm{R}_{\mathrm{DS}(\mathrm{ON})}$ mOhms & $\mathrm{g}_{\mathrm{m}}(\mathrm{S})$ & $\mathrm{I}_{\mathrm{DS}}(\mathrm{A})$ \\
\hline $\mathrm{t}=0$ & 43.04 & 24.88 & 17.01 \\
$\mathrm{~T}=1008$ hours & 54.71 & 22.42 & 13.21 \\
Degradation $(\%)$ & 27.11 & 9.88 & 22.33 \\
\hline
\end{tabular}

Drain current measurements, after each step of aging, highlight the effect of aging of the DUT operating at the limit of the SSOA. The effects are a decrease in the efficiency of DCDC power converters, due to an increase of the power losses of the $\mathrm{DUT}_{\mathrm{M}}$. 
Based on measurements of the aged component, considering the model provided by the constructor lacking precision, an improved SPICE model is defined taking into account the degraded performance of the component. Also, we studied the effect of aging GaN HEMT under SSOA on the efficiency of a DC-DC power converter by a simulation approach. A $12 / 24 \mathrm{~V}$ buck converter is designed which has an output power equals to $180 \mathrm{~W}$ in a load.

The purpose is to model the aging of the $\mathrm{DUT}_{\mathrm{M}}$ in order to evaluate the effects of aging the GaN HEMT under SSOA on the power efficiency of the DC-DC boost converters by a SPICE simulation approach. By applying this methodology, an average decrease of the efficiency of a DC-DC boost converter equals to $4.55 \%$ after aging state for different output current. The decrease in efficiency is accompanied with an increase of conduction power loss in the $\mathrm{DUT}_{\mathrm{M}}$ after aging, the average increase in conduction power losses equals to $8.55 \%$.

\section{Conclusion}

The $\mathrm{SiC}$ and $\mathrm{GaN}$ technologies allow to overcome the limits in the use of energy conversion systems by providing greater energy efficiency, including increased integration and higher switching frequencies.

The challenges are to reduce the size of the power converters and to increase energy efficiency while reducing costs. In the field of transport, the automotive sector, is addressed with the development of hybrid and electric vehicles. The aircraft industry is driven by the demand to optimize aircraft performance, the decrease of operating and maintenance costs and the reduction of gas emissions, are the most demanding sectors of energy efficiency. The field of energy conversion brings the development of the new technology for semiconductor market.

The SiC MOSFET $1200 \mathrm{~V}$ and the GaN $650 \mathrm{~V}$ transistors presented in this paper are two new generation components available on the market. They are accessible to integrators and already offer an alternative to the requirements of integration of energy conversion systems. However, they continue to be the subject of reliability and failure analysis studies, in order to improve their performance and to qualify them in operational conditions for various application sectors.

Acknowledgments. This project is co-funded by European Union and Region Normandy. Europe is involved in Normandy through the European Funds for Regional Development.

\section{References}

[1] Yole Développement. Power SiC : Materials, Devices, Modules and Applications. Technical report, August 2017.

[2] A. J. Lelis, R. Green, D. B. Habersat, and M. El. : Basic mechanisms of threshold voltage instability and implications for reliability testing of sic mosfets. IEEE Transactions on Electron Devices, 62(2) : pp. 316-323, Feb 2015.

[3] V. Mulpuri and S. Choi.: Reliability of sic power mosfets under high repetitive pulse current conditions. IEEE Applied Power Electronics Conference and Exposition (APEC), pages 776-782, March 2017.

[4] Military Standard. Mil-std-750d, test methods for semiconductor devices. Technical report, 1995.

[5] Standard JEDEC. Jesd22a-108 temperature, bias, and operating life.Technical report, 2009. 
[6] Pascal Dherbécourt, Olivier Latry, Karine Dehais-Mourgues, Jean-Baptiste Fonder, CédricDuperrier, Farid Temcamani, HichameMaanane et Jean-Pierre Sipma : Banc de vieillissement de transistors en conditions opérationnelles, Les systèmes mécatroniques embarqués : analyse des causes de défaillances, modélisation, simulation et optimisation, tome 2 Ed ; ISTE (ISBN : 978-178405-059-7) Chap 2, pp. 45-70, Mars 2015.

[7] P. Denis, P. Dherbécourt, O. Latry, C. Genevois, F. Cuvilly, M. Brault, and M. Kadi: Robustness of $4 \mathrm{H}-\mathrm{SiC} 1200 \mathrm{~V}$ schottky diodes under high electrostatic discharge like human body model stresses: An in-depth failure analysis. Diamond and Related Materials, 44:pp62-70, April 2014

[8] WadiaJouha, Ahmed El Oualkadi, Pascal Dherbécourt, Eric Joubert and Mohamed Masmoudi :Silicon Carbide Power MOSFET Model: An Accurate Parameter Extraction Method Based on Levenberg-Marquardt Algorithm», IEEE Transactions on Power electronics, novembre 2018,Volume: 33, Issue:11, pp: 9130-9133

[9] Al Mehdi Bouchour, Andres Echeverri, Olivier Latry, Pascal Dherbécourt, Ahmed El Oualkadi: Modeling of power GaN HEMT for switching circuits applications using Levenberg-Marquardt Algorithm, IEEE International Symposium on Advanced Electrical and Communication Technologies, ISAECT18, 21-23 november 2018, Rabat-Kenitra, Morocco.

[10] SanaeTaoufik, Pascal Dherbécourt, Ahmed El Oualkadi, Member, IEEE, and FaridTemcamani: Reliability and Failure Analysis of UHF RFID Passive Tags under Thermal Storage,IEEE Transactions on Device and Materials Reliability. Volume: 17, Issue: 3, pp. 531-538, September 2017.

[11] ESD STM5.1-2001. Standard Test Method for Electrostatic discharge Sensitivity TestingHuman Body Model (HBM) Component Level. ESD Association WG 5.1, 2001.

[12] ESD STM5.2-1999. Standard Test Method for Electrostatic discharge Sensitivity TestingMachine Model. ESD Association WG 5.2, 1999.

[13] ESD STM5.3.1-1999. Standard Test Method for Electrostatic discharge Sensitivity TestingCharged Device Model (CDM) Component Level. ESD Association, 1999.

[14] ISO 10605. "véhicule routiers : méthodes d'essai des perturbations électriques provenant de décharges électrostatiques". https://www.iso.org, 2001. Accessed : 2018-05-18.

[15] V.A. Vashchenko, Y.B. Martynov, and V.F. Sinkevitch.Electrical instability and filamentation in ggmos protection structures. Solid-State Electronics, 41(11):1761 - 1767, 1997.

[16] M. Sachdev O. Semenov, H. Sarbishaei. ESD Protection Device and Circuit Design for Advanced CMOS Technologies.Springer, Dordrecht, 2008.

[17]Automotive Market Insight, GaN Systems Inc, 2018.

[18]Mitova, Radoslava, et al. "Investigations of $600-\mathrm{V}$ GaN HEMT and GaN diode for power converter applications." IEEE transactions on power electronics 29.5 (2014): 2441-2452.

[19] Lu, Bin, and Tomas Palacios. "High Breakdown $1500 \mathrm{~V}$ AlGaN/GaN HEMTs by SubstrateTransfer Technology." IEEE Electron Device Letters 31.9 (2010): 951-953.

[20] GS66508P Preliminary Datasheet,GaN_Systems_Inc. 2018 